\title{
A CHARACTERIZATION OF PERIODIC AUTOMORPHISMS OF A FREE GROUP
}

BY

\author{
JAMES MCCOOL ${ }^{1}$
}

\begin{abstract}
Let $\theta$ be an automorphism of finite order of a free group $X$. We characterise the action of $\theta$ on $X$ by showing that $X$ has a free basis which is the disjoint union of finite subsets $S_{j}$, where if $S_{j}=\left\{u_{0}, u_{1}, \ldots, u_{k}\right\}$ then $u_{i} \theta=u_{i+1}$ $(0<1<k)$ and $u_{k} \theta=A_{j} u_{0}^{e} B_{j}$ for some $A_{j}, B_{j}$ in $X$ and $\varepsilon= \pm 1$. As an application of this result, we obtain a list of the conjugacy classes of periodic automorphisms of the free group of rank three.
\end{abstract}

1. Introduction. In [2], by using the structure theorem for finite extensions of free groups obtained by Karrass, Pietrowski and Solitar [5], Cohen [1] and Scott [7], Dyer and Scott were able to give an explicit description of the way in which an automorphism of prime order $p$ can act on a free group $X$. The aim of the present work is to extend this result to give a description of the way in which an automorphism $\boldsymbol{\theta}$ of finite order $\boldsymbol{n}$ can act on a free group $X$. Specifically, we shall show that $X$ has a free basis which is the disjoint union of finite subsets $S_{j}$, where if $S_{j}=\left\{u_{0}, u_{1}, \ldots, u_{k}\right\}$ then

$$
u_{i} \theta=u_{i+1} \quad(0<i<k)
$$

and

$$
u_{k} \theta=A_{j} u_{0}^{e} B_{j}
$$

for some $A_{j}, B_{j}$ in $X$ and $\varepsilon= \pm 1$.

Dyer and Scott also showed in [2] that the fixed point subgroup $X^{\pi}$ of any finite group $\pi$ of automorphisms of $X$ is a free factor of $X$. We show, in the case $\pi=\langle\theta\rangle$, that $X^{\langle\theta\rangle}$ has a free basis consisting of the union of all $S_{j}$ above such that $\left|S_{j}\right|=1, A_{j}=B_{j}=1$ and $\varepsilon=1$. We also show, as in the case $n=p$, that $X=$ $X^{\langle\theta\rangle} * H$, where $(H) \theta=H$.

As an application of our main result, we obtain a list of the conjugacy classes of periodic automorphisms of a free group $F$ of rank three. Such a list, in the case where the rank of $F$ is two, has been obtained by Meskin [6], using different techniques.

2. Statement of the result. In order to facilitate the statement of the main theorem, we now introduce the notion of an $n, n_{1}$-based tree. These trees occur as maximal subtrees of certain graphs which arise naturally in the theory of finite extensions of free groups. The reader might find it helpful to examine the second

Received by the editors May 10, 1978 and, in revised form, August 30, 1979.

AMS (MOS) subject classifications (1970). Primary 20E05; Secondary 20 F55.

${ }^{1}$ Research supported by a grant from the National Research Council of Canada. 
half of the proof of the theorem in order to understand the motivation for the introduction of this concept.

Let $\mathcal{T}$ be a tree with a distinguished vertex $a$. By the level $l(c)$ of a vertex $c$ of $\mathcal{T}$, we mean the number of edges in the unique reduced path of $\mathcal{T}$ from $a$ to $c$. Let $n$ be an integer with $n \geqslant 2$, and suppose that:

(1) For each vertex $b$ of $\mathcal{T}$, there are assigned positive integers $\gamma_{b}, \tau_{b}$ with $\gamma_{b}>1$, $\gamma_{b} \tau_{b}=n$ and $\tau_{a}=1$.

(2) For each pair of vertices $c, d$ joined by an edge of $\mathcal{T}$, with $l(c)=l(d)-1$, there are assigned positive integers $\alpha_{c, d}, \beta_{d}$ with $1<\alpha_{c, d}, 1<\beta_{d}, \alpha_{c, d}\left|\gamma_{c}, \beta_{d}\right| \gamma_{d}$ and $\gamma_{c} \beta_{d}=\alpha_{c, d} \gamma_{d}$. We note that $\alpha_{c, d}$ and $\beta_{d}$ depend only on $d$.

(3) For each unordered pair $\{e, f\}$ of vertices with $e \neq f$, a specific order $e, f$ is chosen, satisfying $l(e)<l(f)$, and to each such ordered pair $(e, f)$, and to each pair $(e, e)$, there is assigned a family (possibly empty) of pairs of positive integers $\delta_{e, f, i}$, $\rho_{e, f, i}$, indexed by a set $I_{e, f}$, with $\delta_{e, f, i}\left|\gamma_{e}, \rho_{e, f, i}\right| \gamma_{f}, \gamma_{e} \rho_{e, f, i}=\gamma_{f} \delta_{e, f, i}$, and, in addition, $1<\rho_{e, f, i}, 1<\delta_{e, f, i}$ if $e \neq f$. Also, for each $i \in I_{e, f}$, there is assigned an integer $\eta_{e, f, i}$ satisfying $0 \leqslant \eta_{e, f, i}<\left(\tau_{e}, \tau_{f}\right)$, where $\left(\tau_{e}, \tau_{f}\right)$ is the greatest common divisor of $\tau_{e}$ and $\tau_{f}$

Then $\mathcal{T}$, together with the above assignments, is said to be a based $n, n_{1}$-tree (with base $a$ ) if the least common multiple of the set of all $\beta_{d} \tau_{d}, \delta_{e, f, i} \tau_{e}$ is the integer $n_{1}$. We note that $n_{1}$ is a divisor of $n$.

Our main result is

THEOREM. Let $\mathcal{T}$ be a based $n, n_{1}$-tree with base $a$, as above. Let $X$ be a free group with free generating set $S$, where $S$ is the disjoint union of subsets $S(d)$ (d ranging over the vertices of $\mathcal{T}$ other than $a)$ and subsets $S(e, f, i)(e, f, i$ as in (3) above), with

and

$$
S(d)=\left\{D_{0}, D_{1}, \ldots, D_{\left(\beta_{d}-1\right) \tau_{d}-1}\right\}
$$

$$
S(e, f, i)=\left\{T_{e, f, i, 0}, T_{e, f, i, 1}, \ldots, T_{e, f, i, \rho_{e, f, i} \tau_{f}-1}\right\} .
$$

We shall use the convention that if $d$ is a vertex of $\mathcal{T}$ and $k, j$ are integers with $j>0$ and $k>0$, then $\mathscr{D}_{j}(k)$ shall denote the product $D_{j} D_{j+\tau_{d}} \cdots D_{j+(k-1) \tau_{d}}$

Let $\theta$ be the endomorphism of $X$ defined inductively as follows: firstly,

$$
\begin{aligned}
& D_{k} \theta=D_{k+1} \quad \text { if } 0<k<\left(\beta_{d}-1\right) \tau_{d}-1, \\
& T_{e, f, i, k} \theta=T_{e, f, i, k+1} \quad \text { if } 0<k<\rho_{e, f, i} \tau_{f}-1 ;
\end{aligned}
$$

next, for each vertex $d$ of $\mathcal{T}$ with $d \neq a$, let $c$ be the vertex with $l(c)=l(d)-1$ which is joined to $d$ by an edge of $T$; we define

$$
D_{\left(\beta_{d}-1\right) \tau_{d}-1} \theta=\mathscr{D}_{0}^{-1}\left(\beta_{d}-1\right) \mathcal{C}_{0}\left(\alpha_{c, d}\right)
$$

here we have assumed, inductively, that $C_{i}=C_{0} \theta^{i}$ has been defined for all nonnegative integers $i$, with the convention that, in case $c=a$, we put $A_{i}=1$ for all $i$. This clearly defines $D_{i}=D_{0} \theta^{i}$ for all nonnegative $i$. Finally, we define

$$
T_{e, f, i, \rho_{e, s, i} \tau_{j}-1} \theta=\mathscr{F}_{0}^{-1}\left(\rho_{e, f, i}\right) T_{e, f, i, 0} \mathcal{E}_{\eta_{e, j, i}}\left(\delta_{e, f, i}\right) \text {. }
$$

Then $\theta$ is an automorphism of $X$ of order $n_{1}$.

Conversely, if $\theta$ is an automorphism of order $n$ of a free group $X$, then there is $a$ based $n, n$-tree $\mathcal{T}$ so that $X, \theta$ are defined in terms of $\mathcal{T}$ as above. 
3. Proof of the Theorem. Let $\mathcal{T}$ be a based $n, n_{1}$-tree, and let $X, \theta$ be defined in terms of $\mathcal{T}$ as above. It is easy to see that $(S) \theta$ is a free generating set of $X$, so that $\theta$ is an automorphism of $X$. We now show that $\theta$ has order $n_{1}$.

We note firstly that if $c, d$ are the vertices of an edge of $\mathcal{T}$, with $l(c)=l(d)-1$, then from $\gamma_{c} \tau_{c}=\gamma_{d} \tau_{d}=n$ and $\gamma_{c} \beta_{d}=\alpha_{c, d} \gamma_{d}$, it follows that $\gamma_{c} \tau_{c} \alpha_{c, d}=\gamma_{c} \beta_{d} \tau_{d}$, so that $\alpha_{c, d} \tau_{c}=\beta_{d} \tau_{d}$. Similarly we see that $\delta_{e, f, i} \tau_{e}=\rho_{e, f, i} \tau_{f}$, for all appropriate $e, f, i$.

Next we note that (2.1) may be written as

$$
\mathscr{D}_{0}\left(\beta_{d}\right)=\bigodot_{0}\left(\alpha_{c, d}\right) \text {. }
$$

Now suppose that $d$ is a vertex with $l(d)=1$. Then (3.1) becomes $\mathscr{D}_{0}\left(\beta_{d}\right)=1$. We remark here that if $c$ is a vertex such that $\varrho_{0}(k)=1$ for some $k>0$, and $k_{1}$ is the least positive integer such that $\bigodot_{0}\left(k_{1}\right)=1$, then it is easy to see that $k$ is a multiple of $k_{1}$. Now from $\mathscr{D}_{0}\left(\beta_{d}\right)=\mathscr{D}_{\beta_{d}}\left(\beta_{d}\right)=1$, it follows that $D_{0}=D_{\tau_{d} \beta_{d}}$. Defining the order $m_{d}$ of $\theta$ on $D_{0}$ to be the order of $\theta$ on the subgroup $\left\langle D_{0}, D_{1}, \ldots\right\rangle$ of $X$ generated by all the $D_{i}$, we see that $m_{d} \mid \tau_{d} \beta_{d}$. Now from the definition of $\theta$ we have $m_{d}>\left(\beta_{d}-1\right) \tau_{d}$. Since $\beta_{d}>2$, it follows that $m_{d}=\beta_{d} \tau_{d}$. We note that in this case $m_{d}$ may be described as $\lambda_{d} \tau_{d}$, where $\lambda_{d}$ is the least positive integer such that $\mathscr{D}_{0}\left(\lambda_{d}\right)=1$.

Now let $a, b, \ldots, c, d$ be a reduced path in $\mathcal{T}$ beginning at $a$. Then the subgroup $G$ of $X$ generated by $S(b) \cup \cdots \cup S(c) \cup S(d)$ is clearly invariant under $\theta$. We show

The order of $\theta$ on $G$ is the least common multiple of the set $\left\{\beta_{b} \tau_{b}, \ldots, \beta_{d} \tau_{d}\right\}$

The order $m_{d}$ of $\theta$ on $\mathscr{D}_{0}$ is of the form $\lambda_{d} \tau_{d}$, where $\beta_{d}\left|\lambda_{d}, \lambda_{d}\right| \gamma_{d}$, and $\lambda_{d}$ is the least positive integer such that $\mathscr{D}_{0}\left(\lambda_{d}\right)=1$.

The order of $\theta$ on $G$ is equal to $m_{d}$.

We shall prove these results by induction on the number $q$ of vertices in the path under consideration. Since the results are known if $q=2$, we suppose that $q>2$.

Let $H$ be the subgroup of $G$ generated by $S(b) \cup \cdots \cup S(c)$. Since both $H$ and $G$ are $\theta$ invariant, $\theta$ induces a homomorphism $\theta_{1}$ on $G$ modulo the normal closure of $H$. Applying the argument of the case $l(d)=1$ above to $\theta_{1}$ shows that $m_{d}$ is a multiple of $\tau_{d} \beta_{d}$.

Let $\mu$ be the least common multiple of the pair $\alpha_{c, d}, \lambda_{c}$. We write $\mu=u \alpha_{c, d}=v \lambda_{c}$, and note that $\mu \mid \gamma_{c}$. We have, using the induction hypothesis,

$$
\begin{aligned}
1 & =\bigodot_{0}\left(\lambda_{c}\right) \bigodot_{\lambda_{c} \tau_{c}}\left(\lambda_{c}\right) \cdots \bigodot_{(v-1) \lambda_{c} \tau_{c}}\left(\lambda_{c}\right) \\
& =\bigodot_{0}\left(u \alpha_{c, d}\right) \\
& =\bigodot_{0}\left(\alpha_{c, d}\right) \bigodot_{\alpha_{c, d} \tau_{c}}\left(\alpha_{c, d}\right) \cdots \bigodot_{(u-1) \alpha_{c, d} \tau_{c}}\left(\alpha_{c, d}\right) .
\end{aligned}
$$

Using (3.1) and the fact that $\alpha_{c, d} \tau_{c}=\beta_{d} \tau_{d}$, this becomes

$$
1=\mathscr{D}_{0}\left(\beta_{d}\right) \mathscr{D}_{\beta_{d} \tau_{d}}\left(\beta_{d}\right) \cdots \mathscr{D}_{(u-1) \beta_{d} \tau_{d}}\left(\beta_{d}\right)=\mathscr{D}_{0}\left(u \beta_{d}\right),
$$

and it follows that $m_{d} \mid u \beta_{d} \tau_{d}$. 
Now using $\mathscr{D}_{0}\left(\beta_{d}\right)=\bigodot_{0}\left(\alpha_{c, d}\right)$ and $\mathscr{D}_{\tau_{d}}\left(\beta_{d}\right)=\mathcal{C}_{\tau_{d}}\left(\alpha_{c, d}\right)$ we see that

$$
D_{\beta_{d} \tau_{d}}=\mathcal{C}_{0}^{-1}\left(\alpha_{c, d}\right) D_{0} \mathcal{C}_{\tau_{d}}\left(\alpha_{c, d}\right) \text {. }
$$

Applying $\theta^{(h-1) \beta_{d} \tau_{d}}$ to (3.6), and using induction on $h$, we see that for any positive integer $h$ we have

$$
D_{h \beta_{d} \tau_{d}}=\mathcal{C}_{0}^{-1}\left(h \alpha_{c, d}\right) D_{0} \bigodot_{\tau_{d}}\left(h \alpha_{c, d}\right) .
$$

Now let $m_{d}=h \beta_{d} \tau_{d}$. Since the subgroup of $X$ generated by $S(d)$ and $\left\langle C_{0}, C_{1}, \ldots\right\rangle$ is the free product of these two subgroups, it follows from (3.7) that $\bigodot_{0}\left(h \alpha_{c, d}\right)=1$, so that, by the induction hypothesis, $m_{c}=\lambda_{c} \tau_{c}$ divides $h \alpha_{c, d} \tau_{c}$. Hence $\lambda_{c} \mid h \alpha_{c, d}$. However, since $\mu=u \alpha_{c, d}$ is the least common multiple of $\lambda_{c}$ and $\alpha_{c, d}$, it follows that $u \mid h$. Now $m_{d} \mid u \beta_{d} \tau_{d}$, so that $u=h, m_{d}=u \beta_{d} \tau_{d}$, and (3.3) follows easily, using (3.5).

To prove (3.2) and (3.4) we note that, by the induction hypothesis, $m_{c}=$ $\operatorname{lcm}\left\{\beta_{b} \tau_{b}, \ldots, \beta_{c} \tau_{c}\right\}=\lambda_{c} \tau_{c}$. Now

$$
\begin{aligned}
\operatorname{lcm}\left\{\beta_{b} \tau_{b}, \ldots, \beta_{d} \tau_{d}\right\} & =\operatorname{lcm}\left\{l_{c} \tau_{c}, \beta_{d} \tau_{d}\right\}=\operatorname{lcm}\left\{\lambda_{c} \tau_{c}, \alpha_{c, d} \tau_{c}\right\} \\
& =\tau_{c} \operatorname{lcm}\left\{\lambda_{c}, \alpha_{c, d}\right\}=\tau_{c} u \alpha_{c, d} \\
& =u \beta_{d} \tau_{d}=m_{d}
\end{aligned}
$$

as required.

We now investigate the order $m=m_{e, f, i}$ of $\theta$ on the generator $T_{e, f, i, 0}$. For ease of notation, we shall omit the subscripts $e, f, i$ from $T_{e, f, i, j}, \delta_{e, f, i}, \rho_{e, f, i}$, and $\eta_{e, f, i}$. We have $S(e, f, i)=\left\{T_{0}, T_{1}, \ldots, T_{\rho r_{j}-1}\right\}$, and, writing $T_{j}$ for $T_{0} \theta^{j}$ for all nonnegative $j$, (2.2) becomes

$$
T_{\rho \tau_{f}}=\mathscr{F}_{0}^{-1}(\rho) T_{0} \mathcal{E}_{\eta}(\delta) .
$$

From this, it is easily shown that for any $k>1$ we have

$$
T_{k \rho \tau_{f}}=\mathscr{F}_{0}^{-1}(k \rho) T_{0} \mathcal{E}_{\eta}(k \delta) \text {. }
$$

Now factoring out the subgroup of $X$ generated by the union of the sets $S(d)$ shows immediately that $m$ is a multiple of $\rho \tau_{f}$. It then follows from (3.9) that $m=k \rho \tau_{f}$, where $k$ is the least positive integer such that $\mathscr{F}_{0}(k \rho)=1$ and $\delta_{0}(k \delta)=$ 1. Thus $k$ is the least positive integer such that $\lambda_{f} \mid k \rho$ and $\lambda_{e} \mid k \delta$, i.e. such that $m_{f} \mid k \rho \tau_{f}$ and $m_{e} \mid k \delta \tau_{e}$. Hence $m=\operatorname{lcm}\left\{m_{e}, m_{f}, \rho \tau_{f}\right\}$.

It now follows immediately that the order of $\theta$ on $X$ is the least common multiple of the set of all $\beta_{d} \tau_{d}$ and $\delta_{e, f} \tau_{e}$, i.e. $|\theta|=n_{1}$. This proves the first part of the Theorem.

Now we suppose that $\theta$ is an automorphism of order $n$ of a free group $X$. We show that there is a based $n, n_{1}$-tree $\mathcal{T}$ so that $X, \theta$ are described in terms of $\mathcal{T}$ as in the statement of the Theorem. The approach used is similar to that of [2], and as in [2], we shall use the terminology of [1] and [8].

We denote by $Y$ the split extension of $X$ by $Z_{n}$, a cycle of order $n$, formed using the action of $\theta$ on $X$, and by $\pi$ the natural projection from $Y$ to $Z_{n}$ with kernel $X$. The fundamental theorem of Karrass et al. ([5], [1], [7]) tells us that $Y$ is an HNN extension whose base is a tree product of finite groups, and whose associated 
subgroups lie in vertex groups of the base. In the language of [8], this states that $Y$ is the fundamental group of a connected graph of groups $\mathcal{Y}$, which is such that every vertex group $Y_{d}$ of $\mathcal{Y}$ is finite.

Since $X$ is torsion-free, $\pi$ provides an injection of each finite subgroup of $Y$ into $Z_{n}$. Thus each finite subgroup of $Y$ is cyclic, and of order a divisor of $n$.

From the construction of $Y$, there exists $A \in Y$ such that $\langle A\rangle$ has order $n$, and $A x A^{-1}=(x) \theta$, for all $x \in X$. By conjugating $\mathcal{Y}$ (see [1]) if necessary, we may assume that $A$ is in a vertex group $Y_{a}$ of $\mathscr{Y}$. It then follows that $Y_{a}=\langle A\rangle$.

We now observe that by applying suitable contractions (see [1]) to $\mathcal{Y}$, we may assume, without loss of generality, that for each edge $v$ of $\mathcal{Y}$ which is not a loop, the associated subgroup of $v$ is (embedded as) a proper subgroup of each of the two corresponding vertex groups.

Now let $\mathcal{T}$ be a maximal subtree of (the graph of) $\mathcal{Y}$, regarded as being based at $a$. For each pair of vertices $e, f$ with $e \neq f$ we choose an order $e, f$ as in part (3) of the definition of a based $n$-tree. For such an ordered pair $e, f$ the (directed) edges of $\mathcal{Y}$, not in $\mathcal{T}$, joining $f$ to $e$ will be called free edges of $\mathcal{Y}$, and are regarded as being indexed by a set $I_{e, f}$. Also, for each vertex $e$ and each (undirected) edge beginning and ending at $e$, we select one of the two corresponding directed edges. The edges so selected are also called free edges of $\mathcal{Y}$, and those at vertex $e$ are regarded as being indexed by the set $I_{e, e}$. It now follows (see [8]) that $Y$ has presentation $\mathscr{P}=(\mathcal{S} ; \Re)$, where $\mathcal{S}$ consists of one generator $D$ for each vertex $d$ of $\mathscr{Y}(D$ corresponds to a generator of the vertex subgroup $Y_{d}$ of $Y$ ) and one generator $T_{e, j, i}$ for each $i \in I_{e f}$, where $(e, f)$ ranges over the selected pairs of edges (the $T$ generators correspond to the free part of the HNN extension $Y$ ). Here $\mathcal{R}$ consists of three sets of relations; firstly the set $\Re_{1}$, consisting of one relation $D^{\gamma_{d}}=1$ for each vertex generator, where $\gamma_{d}$ is the order of the vertex subgroup $Y_{d}$, so that

$$
1<\gamma_{d} \leqslant n, \quad \gamma_{d} \mid n, \quad \gamma_{a}=n .
$$

Next, $\Re_{2}$ consists of one relation $C^{\alpha_{r, d}}=D^{\beta_{d}}$ for each edge $v$ of $\mathcal{T}$, where $c, d$ are the vertices of $v$, and $l(c)=l(d)-1$. This relation corresponds to the embedding of the associated subgroup of $v$ as a subgroup of $Y_{c}$ and $Y_{d}$, so that

$$
1<\alpha_{c, d}, \quad 1<\beta_{d}, \quad \gamma_{c} /\left(\alpha_{c, d}, \gamma_{c}\right)=\gamma_{d} /\left(\beta_{d}, \gamma_{d}\right) .
$$

In addition, we may assume that

$$
\alpha_{c, d} \mid \gamma_{c}
$$

since the subgroup $\left\langle C^{\alpha_{r, d}}\right\rangle$ has a generator $C^{k}$ with $k \mid \gamma_{c}$, and it is sufficient to identify $C^{k}$ with an appropriate power of $D$.

Finally, $\mathscr{R}_{3}$ consists of one relation

$$
T_{e, f, i} E^{\delta_{e s . i}} T_{e f, i}^{-1}=F^{\rho_{e f, i}}
$$

for each 'free generator' $T_{e, f, i}$ of $Y$. These are the HNN relations of $Y$, and correspondingly we have

$$
1 \leqslant \delta_{e, f, i}, \quad 1<\rho_{e_{e}, i}
$$


with strict inequality if $e \neq f$, and

$$
\gamma_{e} /\left(\gamma_{e}, \delta_{e, f, i}\right)=\gamma_{f} /\left(\gamma_{f}, \rho_{e, f, i}\right)
$$

In addition, we may assume that

$$
\delta_{e, f, i} \mid \gamma_{e}
$$

Now from the choice of $A$, it follows that for each $D$ generator there is a positive integer $\tau_{d}$ such that $D \pi=(A \pi)^{\tau_{d}}$, i.e. that $D A^{-\tau_{d}} \in X$. Since $A \pi$ has order $n$, it follows that $\gamma_{d}=n /\left(n, \tau_{d}\right)$. Let $r, s$ be integers such that $r \tau_{d}+s n=\left(n, \tau_{d}\right)$. Then $\left(r, \gamma_{d}\right)=1$, so that $\langle D\rangle=\left\langle D^{r}\right\rangle$. We note that $D^{r} \pi=(A \pi)^{\tau_{d} r}=(A \pi)^{\left(n, \tau_{d}\right)}$. Clearly we could have selected the generator $D^{r}$ originally, in place of $D$, when obtaining the presentation $\mathcal{P}$. Thus we may assume that $D$ was chosen so that

$$
\gamma_{d} \tau_{d}=n \text {. }
$$

The conditions (3.10) to (3.15) continue to hold with this choice of $D$.

We now show that $\beta_{d}$ may be chosen so that $\beta_{d} \mid \gamma_{d}$. Applying $\pi$ to the relations in $\Re_{2}$ we obtain, since $(Y) \pi$ is abelian, that

so that

$$
(A \pi)^{\tau_{c} \alpha_{c, d}}=(A \pi)^{\tau_{d} \beta_{d}},
$$

$$
\tau_{c} \alpha_{c, d} \equiv \tau_{d} \beta_{d}(\bmod n)
$$

From (3.10) and (3.11) we have $\alpha_{c, d}=\gamma_{c}\left(\beta_{d}, \gamma_{d}\right) / \gamma_{d}$, and substitution in (3.17) yields

$$
\tau_{c} \gamma_{c}\left(\beta_{d}, \gamma_{d}\right) / \gamma_{d} \equiv \tau_{d} \beta_{d} \quad(\bmod n) .
$$

Now from (3.16) we have $\tau_{c} \gamma_{c}=\tau_{d} \gamma_{d}$, so that (3.18) may be written as

$$
\tau_{d}\left(\beta_{d}, \gamma_{d}\right) \equiv \tau_{d} \beta_{d}(\bmod n)
$$

and therefore

$$
\left(\beta_{d}, \gamma_{d}\right) \equiv \beta_{d} \quad\left(\bmod n / \tau_{d}=\gamma_{d}\right) .
$$

Since $D$ has order $\gamma_{d}$, it follows that $D^{\beta_{d}}=D^{\left(\beta_{d}, \gamma_{d}\right)}$. Thus we may assume $\beta_{d} \mid \gamma_{d}$.

Now by applying $\pi$ to $\mathscr{R}_{3}$, we see that

$$
\tau_{e} \delta_{e, f, i} \equiv \tau_{f} \rho_{e, f, i}(\bmod n) .
$$

It is now easy to see, by an argument similar to that used in the case for $\Re_{2}$, that $\rho_{e, f, i} \mid \gamma_{f}$.

For each free generator $T_{e, f, i}$ we have $\left(T_{e, f, i}\right) \pi=(A \pi)^{\eta_{S, 1, i}}$, for some integer $\eta_{e, f, i}$ satisfying $0<\eta<n$. Since $T_{e, f, i}$ occurs in only one relation of $\mathcal{P}$, we may, in view of the form of that relation, replace $T_{e, f, i}$ by a new generator $T_{e, f, i}^{\prime}$, where $T_{e, f, i}^{\prime}=$ $F^{r} T_{e, f, i} E^{s}$ for some integers $r, s$, and the presentation obtained is just $\mathscr{P}$ again, if we now denote $T_{e, f, i}^{\prime}$ by $T_{e, f, i}$. We then have

$$
\left(T_{e, f, i}\right) \pi=(A \pi)^{\pi_{f}+s T_{e}+\eta_{e s, i} .}
$$

We choose $r, s$ so that $r \tau_{f}+s \tau_{e}+\eta_{e, f, i}$ is the remainder on division of $\eta_{e, f, i}$ by $\left(\tau_{e}, \tau_{f}\right)$. Thus we may suppose that $0 \leqslant \eta_{e, f i}<\left(\tau_{e}, \tau_{f}\right)$.

It follows from the above that $\mathcal{T}$ is a based $n, n_{1}$-tree, where $n_{1}$ is the least common multiple of the set of all $\beta_{d} \tau_{d}, \delta_{e, f, i} \tau_{e}$. 
We now apply the Reidemeister-Schreier rewriting process (cf. [5]) to obtain a presentation for $X$. We use the coset representative system $1, A, \ldots, A^{n-1}$ for $X$ in $Y$. Writing $D_{i}$ for the word $A^{i} D A^{-\left(\tau_{d}+i\right)^{\prime}}$, where $0<i<n$ and $\left(\tau_{d}+i\right)^{\prime}$ is the remainder of $\tau_{d}+i$ on division by $n$, and similarly writing $T_{e, f, i j}$ for $A^{j} T_{e_{j}, i} A^{-\left(j+\eta_{e, j, i}\right)^{\prime}}$, where $0 \leqslant j<n$, we find that $X$ is generated by all the $D_{i}$ and $T_{e, f, i j}$, together with $W=A^{n}$. Rewriting the relations of $Y$ in terms of these generators, we firstly obtain $W=1$ from the relation $A^{n}=1$. We shall therefore delete all occurrences of $W$ in what follows.

Rewriting $A^{i} D^{\gamma_{d}} A^{-i}(d \neq a)$ we have, if $i=k \tau+s$, where $\tau=\tau_{d}, 0<s<\tau$ and $\gamma=\gamma_{d}$, that

$$
\begin{aligned}
A^{i} D^{\gamma_{d}} A^{-i}= & \left\{A^{k \tau+s} D A^{-\tau(k+1)-s}\right\}\left\{A^{\tau(k+1)+s} D A^{-\tau(k+2)-s}\right\} \cdots \\
& \left\{A^{\tau(\gamma-1)+s} D A^{-s}\right\}\left\{A^{s} D A^{-\tau-s}\right\} \cdots\left\{A^{\tau(k-1)} D A^{-\tau k-s}\right\} .
\end{aligned}
$$

Thus we obtain from $\Re_{1}$ the following set of relations of $X$ :

$$
D_{s} D_{s+\tau_{d}} \cdots D_{s+\left(\gamma_{d}-1\right) \tau_{d}}=1 \text {, }
$$

where $0<s<\tau_{d}$. In our previous notation, these relations may be written as

$$
\mathscr{Q}_{s}\left(\gamma_{d}\right)=1
$$

for $0<s<\tau_{d}$.

A similar argument shows that the relations $\mathscr{R}_{2}$ yield the following set of relations for $X$, where $0 \leqslant j<n-1$ :

$$
\bigodot_{j}\left(\alpha_{c, d}\right)=\mathscr{D}_{j}\left(\beta_{d}\right)
$$

with the understanding that the subscripts in the written out forms of $\mathcal{C}_{j}\left(\alpha_{c, d}\right)$ and $\mathscr{D}_{j}\left(\beta_{d}\right)$ are to be reduced modulo $n$, and in case $c=a$ we put $A_{j}=1$ for all $j$.

Now we rewrite the word $T E^{\delta} T^{-1} F^{-\beta}$ corresponding to the relation $\Re_{3}$, where we have omitted the subscripts $e, f, i$. We may assume that $\delta \tau_{e}<n$, since otherwise the relation is a consequence of $\Re_{1}$. We have

$$
T E^{\delta} T^{-1} F^{-\rho}=\left(T A^{-\eta}\right)\left(A^{\eta} E A^{-\eta-\tau_{e}}\right) \cdots\left(A^{\eta+(s-1) \tau_{e}} E A^{-\eta-\delta \tau_{e}}\right)\left(A^{\eta+s \tau_{e}} T^{-1} F^{-\rho}\right) .
$$

We note that $\eta+\delta \tau_{e}<n$, since $\delta \tau_{e} \mid n, \delta \tau_{e}<n$ and $\eta<\tau_{e}$. Rewriting $F^{\rho} T A^{\eta+\delta r_{e}}$ we have, since $\delta \tau_{e}=\rho \tau_{f}$,

$$
F^{\rho} T A^{-\eta-\delta \tau_{e}}=\left(F A^{-\tau_{f}}\right)\left(A^{\tau_{f}} F A^{-2 \tau_{f}}\right) \cdots\left(A^{(\rho-1) \tau_{f}} F A^{-\rho \tau_{f}}\right)\left(A^{\rho \tau_{f}} T A^{-\eta-\rho \tau_{f}}\right) .
$$

Thus the corresponding relation of $X$ is

$$
T_{0} E_{\eta} E_{\eta+\tau_{e}} \cdots E_{\eta+(s-1) \tau_{e}}=F_{0} F_{\tau_{f}} \cdots F_{(\rho-1) \tau_{j}} T_{\rho \tau \rho}
$$

which may be written, with the same understanding as in the case of $\mathscr{R}_{2}$, in the form $T_{0} \mathcal{E}_{\eta}(\delta)=\mathscr{F}_{0}(\rho) T_{\rho \tau_{j}}$ A similar argument shows that the relation of $X$ corresponding to the rewritten word $A^{j} T E^{\delta} T^{-1} F^{-\rho} A^{-j}$, where $0<j<n$, is

$$
T_{j} \mathcal{E}_{j+\eta}(\delta)=\mathscr{F}_{j}(\rho) T_{j+\rho \tau_{j}}
$$

and this is therefore the set of relations of $X$ corresponding to the relations $\Re_{3}$, where we have omitted the subscripts $e, f, i$.

Now let $X_{1}, \theta_{1}$ be the free group and corresponding automorphism constructed from the based $n, n_{1}$-tree $\mathcal{T}$ as in the statement of the Theorem. We regard $X_{1}$ as 
being generated by the set $S_{1}$ of all $D_{0}, D_{1}, \ldots, D_{n-1}$ and $T_{0}, T_{1}, \ldots, T_{n-1}$. We note that, since $n_{1} \mid n$, the elements of $S_{1}$ continue to satisfy the relations given by (3.1) and (3.8), when the subscripts in these are reduced modulo $n$. These relations are clearly sufficient to express each element of $S_{1}$ as a word on the free generating set $S$ of $X_{1}$, and hence constitute a set of defining relations for $X_{1}$ on the generating set $S_{1}$. In addition, it follows from (3.3) that the set of relations $\mathscr{D}_{0}\left(\gamma_{d}\right)=1$, with reduced subscripts, are satisfied by the $D$-generators of $X_{1}$. Comparing with (3.19), (3.20) and (3.21), we see that $X$ and $X_{1}$ have the same presentation on the set $S_{1}$, and that the actions of $\theta$ and $\theta_{1}$ are the same. It follows that $n=n_{1}$ and that $X, \theta$ have the required form. This proves the Theorem.

4. Remarks on the Theorem. Let $X, \theta$ be defined as in the statement of the Theorem, and let $\mathcal{O}$ be the corresponding graph of groups. We have:

(1) If $X$ is finitely generated then it follows immediately from the definition of $X$ that its rank $r(X)$ is given by

$$
r(X)=\sum_{d}\left(\beta_{d}-1\right) \tau_{d}+\sum_{e, f, i} \rho_{e, f, i} \tau_{f}
$$

(2) It is easy to see, from the conditions on the various parameters used, that the description of the action of $\theta$ on $X$ reduces to that of [2] in the case $n$ is a prime.

(3) We now find the fixed point subgroup $X^{\langle\theta\rangle}$ of $\theta$. We note that if $K$ is the centralizer of $A$ in $Y$, then $X^{\langle\theta\rangle}$ is the intersection of $K$ and $X$.

Let $T=T_{a, a, i}$ be a 'free generator' of $Y$ such that $\rho_{a, a, i}=1$. Then $T \in K$, and if $K_{1}$ is the group generated by $A$ and all such $T$, we shall show that $K=K_{1}$.

To prove this, we use the normal form theorem for the elements of the fundamental group of a graph of groups which was given in [3]. We begin with some notation. Let $r$ be a (directed) edge of $\mathcal{Y}$, with initial vertex $e$ and terminal vertex $f$. We denote by $L(r)$ the subgroup of $Y_{e}$ associated with $r$, and by $M(r)$ the corresponding subgroup of $Y_{f}$. Of course, if $\bar{r}$ denotes the edge opposite to $r$, then $L(\bar{r})=M(r)$. We now choose, for each $r$, a coset representative system $\mathcal{L}(r)$ for $L(r)$ in $Y_{e}$, so that $Y_{e}=\mathcal{L}(r) L(r)$, and $1 \in \mathcal{L}(r)$. We now define an element $t_{r}$ of $Y$ for each edge $r$. If $r \in \mathcal{T}$, then we put $t_{r}=1$; otherwise, we put $t_{r}=T_{e, f, i}^{-1}$ or $t_{r}=T_{e, i, i}$, according as $r$ or $\bar{r}$ is the $i$ th free edge corresponding to the pair of vertices $e, f$ (as in the construction of $\mathcal{T}$ in the second part of the proof of the Theorem).

The normal form theorem of [3] (see also [9]) states that every nonidentity element $y$ of $Y$ has a unique expression

$$
y=\gamma_{1} t_{r_{1}} \gamma_{2} t_{r_{2}} \cdots \gamma_{k} t_{r_{k}} h
$$

where $k \geqslant 0, r_{1}, r_{2}, \ldots, r_{k}$ are the successive edges of a path in $\mathcal{Y}$ beginning and ending at $a, \gamma_{j} \in \mathcal{L}\left(r_{j}\right), 1 \leqslant j \leqslant k, h \in Y_{a}$ and $r_{j-1} \neq \bar{r}_{j}$ if $\gamma_{j}=1(2<j<k)$.

We note that if the edge $r$ has initial vertex $a$, then we may suppose that the transversal $\mathcal{L}(r)$ contains $A$, unless $r$ is a loop at $a$ with corresponding $\rho=1$, since if $r$ is not such a loop then the subgroup $L(r)$ is a proper subgroup of $\langle A\rangle$.

Let $y \in Y$, with normal form (4.2), be an element of $K$. We show that $y \in K_{1}$. We may suppose that $k \geqslant 1$, since otherwise $y=h \in K_{1}$. We note that $\gamma_{1} \in K_{1}$, 
and that replacing $\gamma_{1}$ by 1 in the right side of (4.2) again yields a normal form expression. Thus we may suppose that $\gamma_{1}=1$. Next, if $t_{r_{1}}$ is a loop at $a$ with corresponding $\rho=1$, then $t_{r_{1}} \in K_{1}$, and the expression obtained from the right side of (4.2) by deleting $\gamma_{1} t_{r_{1}}$ is again a normal form expression. Thus we may suppose, also, that $r_{1}$ is not such a loop. We show that, with these assumptions, we cannot have $k>1$. Suppose otherwise. Then, from the conditions satisfied by $\gamma_{1}$ and $r_{1}$, it follows immediately that both $A t_{r_{1}} \gamma_{2} \cdots t_{r_{k}} h$ and $\gamma_{1} t_{r_{1}} \cdots t_{r_{k}}(h A)$ are normal form expressions. Since these expressions are both equal to $y$, they must be identical. This is clearly not the case, and hence $k=0$. This proves that $K=K_{1}$.

It follows that $X^{\langle\theta\rangle}$ is the subgroup of $X$ generated by all $T_{a, a, i, 0}$ with $\rho_{a, a, i}=1$. Visibly, $X^{\langle\theta\rangle}$ is a free factor of $X$, and $X=X^{\langle\theta\rangle} * H$, where $H \theta=H$.

5. The periodic automorphisms of the free group of rank three. The Theorem can be used to verify that each periodic automorphism of a free group of rank three is conjugate to exactly one of the following: First, we have eight automorphisms of order two

$$
\begin{array}{lll}
B_{0} \rightarrow B_{0}^{-1}, & C_{0} \rightarrow C_{0}^{-1}, & D_{0} \rightarrow D_{0}^{-1}, \\
B_{0} \rightarrow B_{0}^{-1}, & C_{0} \rightarrow C_{0}^{-1}, & T_{0} \rightarrow T_{0}, \\
B_{0} \rightarrow B_{0}^{-1}, & T_{0} \rightarrow T_{0}, & J_{0} \rightarrow J_{0}, \\
B_{0} \rightarrow B_{0}^{-1}, & C_{0} \rightarrow C_{0}^{-1}, & T_{0} \rightarrow B_{0}^{-1} T_{0} B_{0}, \\
B_{0} \rightarrow B_{0}^{-1}, & T_{0} \rightarrow T_{1} \rightarrow T_{0}, & \\
B_{0} \rightarrow B_{0}^{-1}, & T_{0} \rightarrow B_{0}^{-1} T_{0} B_{0}, & J_{0} \rightarrow J_{0}, \\
B_{0} \rightarrow B_{0}^{-1}, & T_{0} \rightarrow B_{0}^{-1} T_{0} B_{0}, & J_{0} \rightarrow B_{0}^{-1} J_{0} B_{0}, \\
T_{0} \rightarrow T_{1} \rightarrow T_{0}, & J_{0} \rightarrow J_{0} . &
\end{array}
$$

Next, we have three automorphisms of order three

$$
\begin{array}{ll}
B_{0} \rightarrow B_{1} \rightarrow B_{1}^{-1} B_{0}^{-1}, & T_{0} \rightarrow T_{0}, \\
B_{0} \rightarrow B_{1} \rightarrow B_{1}^{-1} B_{0}^{-1}, & T_{0} \rightarrow B_{0}^{-1} T_{0} B_{0}, \\
T_{0} \rightarrow T_{1} \rightarrow T_{2} \rightarrow T_{0} . &
\end{array}
$$

Next, we have three automorphisms of order four

$$
\begin{aligned}
& B_{0} \rightarrow B_{1} \rightarrow B_{0}^{-1}, \quad C_{0} \rightarrow C_{0}^{-1}, \\
& B_{0} \rightarrow B_{1} \rightarrow B_{0}^{-1}, \quad T_{0} \rightarrow T_{0}, \\
& B_{0} \rightarrow B_{1} \rightarrow B_{2} \rightarrow B_{2}^{-1} B_{1}^{-1} B_{0}^{-1} .
\end{aligned}
$$

Finally, we have four of order six.

$$
\begin{array}{ll}
B_{0} \rightarrow B_{1} \rightarrow B_{1}^{-1} B_{0}^{-1}, & C_{0} \rightarrow C_{0}^{-1}, \\
B_{0} \rightarrow B_{1} \rightarrow B_{1}^{-1} B_{0}^{-1}, & C_{0} \rightarrow C_{0}^{-1} B_{0} B_{1}, \\
B_{0} \rightarrow B_{1} \rightarrow B_{1}^{-1} B_{0}^{-1} C_{0}, & C_{0} \rightarrow C_{0}^{-1}, \\
B_{0} \rightarrow B_{1} \rightarrow B_{2} \rightarrow B_{0}^{-1} . &
\end{array}
$$


We indicate briefly how the list is obtained. Let $X$ be a free group of rank three, $\theta$ a periodic automorphism of $X, \mathcal{Y}$ the corresponding graph of groups and $\mathcal{T}$ a maximal tree in $\mathcal{Y}$. Since each $\beta_{d}>2$, it follows from (4.1) that the rank of $X$ is at least $\Sigma_{c} \tau_{c}$, where $c$ ranges over the vertices of $\mathcal{O}$ other than $a$. Thus $\mathcal{Y}$ has at most four vertices. It is now a simple matter to list all possible graphs $\mathcal{O}$ and compute the corresponding automorphisms. To illustrate this, suppose that $\mathcal{O}$ has three vertices $a, b, c$. From (4.1) we see that there are two possibilities for the pair $\tau_{b}, \tau_{c}$, namely $\tau_{b}=\tau_{c}=1$ and $\tau_{b}=2, \tau_{c}=1$. We consider only the first case (the second gives rise to the automorphism (5.12)). For the case $\tau_{b}=\tau_{c}=1$ we may have, again from (4.1), either $\beta_{b}=\beta_{c}=2$ and $\mathcal{Y}$ has one free edge with $\rho=1$ (and the free edge must therefore be a loop, with $\eta$ value zero since all $\tau$ values are one) or $\beta_{b}=3, \beta_{c}=2$ and $\mathcal{Y}$ has no free edges. We consider only the latter possibility (the former gives rise to the automorphisms (5.2) and (5.4)).

Thus we have vertices $a, b, c$ with $\tau_{b}=\tau_{c}=1, \beta_{b}=3, \beta_{c}=2$. Hence $|\theta|=6$, and all $\gamma$ values are 6 . There are three possible labellings of the vertices of the tree $\mathcal{Y}$, from which we obtain three (isomorphic) groups, namely $\left\langle A, B, C ; A^{6}=B^{6}=\right.$ $\left.C^{6}=1, A^{3}=B^{3}, A^{2}=C^{2}\right\rangle$, and the groups obtained from this by, firstly, interchanging $A$ and $B$, and, secondly, interchanging $A$ and $C$. The $\alpha$ values can now be read from this, and the automorphisms obtained are, respectively, (5.15), (5.16) and (5.17).

On obtaining the list of possible $\theta$ 's, as given, some further arguments are required to show that no two of the elements listed are conjugate. Details are available from the author.

\section{REFERENCES}

1. D. E. Cohen, Groups with free subgroups of finite index, Conference on Group Theory, Lecture Notes in Math., vol. 319, Springer, Berlin, 1973, pp. 26-44.

2. J. L. Dyer and G. P. Scott, Periodic automorphisms of free groups, Comm. Algebra 3 (1975), 195-201.

3. P. J. Higgins, The fundamental group of a graph of groups, J. London Math. Soc. 13 (1976), 145-149.

4. W. Magnus, A. Karrass and D. Solitar, Combinatorial group theory, Wiley (Interscience), New York, 1966.

5. A. Karrass, A. Pietrowski and D. Solitar, Finitely generated groups with a free subgroup of finite index, J. Austral. Math. Soc. 16 (1973), 458-466.

6. S. Meskin, Periodic automorphisms of the two-generator free group, Conference on Group Theory, Lecture Notes in Math., vol. 372, Springer, Berlin, 1974, pp. 494-498.

7. G. P. Scott, An embedding theorem for groups with a free subgroup of finite index, Bull. London Math. Soc. 6 (1974), 304-306.

8. J. P. Serre, Arbres, amalgames, $S L_{2}$, Astérisque 46, Société Mathématique de France, 1977.

9. M. D. Tretkoff, A topological approach to HNN extensions and the theory of groups acting on trees, preprint.

Department of Mathematics, University of Toronto, Toronto M5S 1A1, Ontario, Canada 